\title{
ПРОБЛЕМЫ ПРОВЕДЕНИЯ ИЗМЕРЕНИЙ ОБЛУЧЕННОСТИ В ПРОЦЕССЕ ЛАЗЕРНОЙ ОБРАБОТКИ МЕТАЛЛОВ
}

\author{
O.A. Крючина, оKryuchina@ntoire-polus.ru, \\ И. Э.Садовников, iSadovnikov@ntoire-polus.ru, \\ ООО НТО «ИРЭ-Полюс», Фрязино, Моск. обл.
}

\begin{abstract}
Реализованный в дозиметре метод контроля облученности от рассеянного и отраженного лазерного излучения в процессе лазерной обработки металлов подразумевает измерения облученности пространства рабочего места оператора только в процессах с неподвижным пучком. Современные лазерные технологии базируются на высокоскоростных движениях лазерных пучков, оттого ранее разработанные методы контроля параметров лазерной облученности требуют принципиально новых подходов к методам дозиметрического контроля. В статье представлены данные об особенностях проведения измерений облученности отраженного и рассеянного лазерного излучения при обработке металлов подвижным лазерным пучком и рассматриваются возможные пути решения возникших проблем.
\end{abstract}

\section{ВВЕДЕНИЕ}

Стремительное развитие лазерной техники и лазерных технологий, появление роботизированных комплексов, а также их активное внедрение во многие сферы деятельности человека позволяет говорить о том, что лазерная индустрия вышла на принципиально новый уровень.

В настоящее время в НТО "ИРЭ-Полюс" интенсивно разрабатывают и серийно производят мощные волоконные лазеры, создана основа их широкого применения в составе технологических установок (резка, маркировка, сварка, наплавка, и т.д.) в разных отраслях промышленности, а также в медицинском оборудовании.

При проведении измерений лазерным дозиметром (ЛД) облученности рассеянного и отраженного лазерного излучения (ЛИ) в процессе лазерной обработки металлов приходится сталкиваться с рядом вопросов и проблем.

Наиболее востребованное средство измерений, внесенное в Госреестр, - дозиметр «ЛД-07» (рис. 1). ЛД является переносным прибором и состоит из блока фотоприемников (БФП) (поз. 2 на рис. 1) и блока управления и индикации (БУИ) (поз. 1 на рис. 1). Данные измерений обрабатываются встроенным в прибор микропроцессором по заложенной программе. Результаты измерений высвечиваются на жидкокристаллическом дисплее БУИ.
Прибор обеспечивает:

- регистрацию и учет фоновых показателей (облученности $E_{\phi}\left[\mathrm{BT} / \mathrm{cm}^{2}\right]$, энергетической экспозиции $\mathrm{H}_{\phi}\left[\right.$ Дж/ $\left.\left.\mathrm{cm}^{2}\right]\right)$;

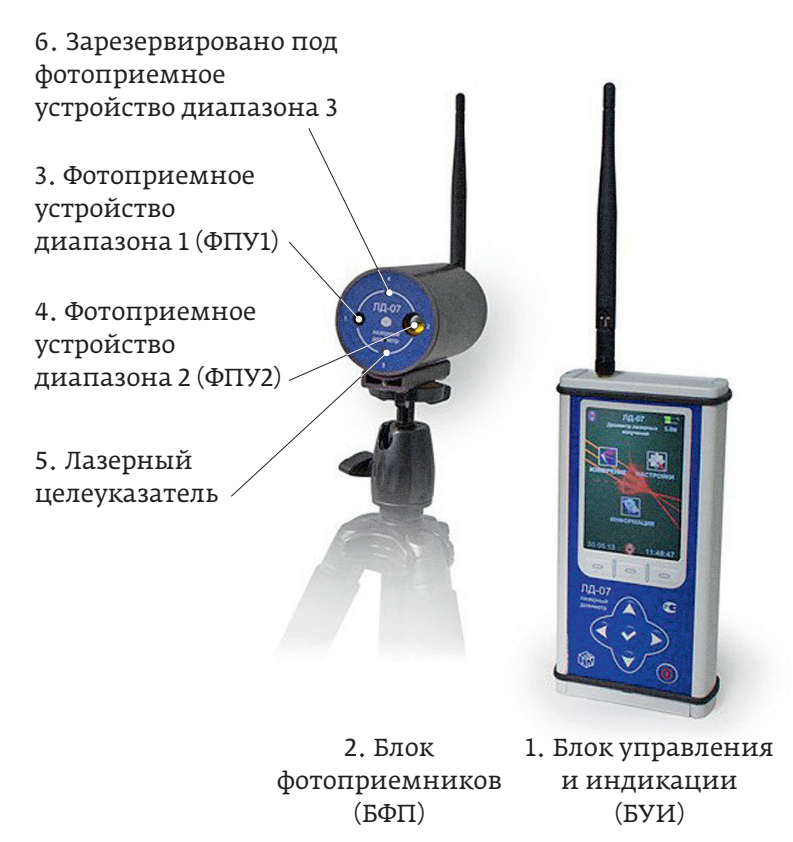

Рuc. 1. Лазерный дозиметр «ЛД-07» 
- регистрацию наибольшего значения измеряемого параметра (облученности $E_{\max }\left[\mathrm{BT} / \mathrm{cm}^{2}\right]$ и энергетической экспозиции $\mathrm{H}_{\max }$ [Дж/ $\left.\mathrm{cm}^{2}\right]$ ) ли за цикл измерений;

- измерение текущих значений параметров (облученности $E\left[\mathrm{BT} / \mathrm{cm}^{2}\right]$, энергетической экспозиции $\mathrm{H}\left[\right.$ [Жж/ $\left.\mathrm{cm}^{2}\right]$ ) рассеянного или отраженного Ли [1].

\section{УЧЕТ ФОНОВЫХ ПОКАЗАТЕЛЕЙ ОБЛУЧЕННОСТИ ЛИ}

Учет фоновых показателей осуществляется путем обнуления показаний нажатием по иконке "0". Данная функция очень актуальна при проведении измерений в производственных условиях, т. к. уровень фоновых значений меняется с достаточно большой частотой. Поскольку прибор не обладает селективностью по длине волны, то любое изменение естественного или искусственного освещения оказывает влияние на показания прибора. Это критично при получении малых значений облученности, когда значения $E_{\max }$ могут быть ниже уровня фона. На эту особенность надо обратить внимание при измерениях и по возможности учитывать фоновые значения перед проведением каждого измерения.

\section{РЕГИСТРАЦИЯ НАИБОЛЬШЕГО ЗНАЧЕНИЯ ОБЛУЧЕННОСТИ ЛИ}

Измерения $E_{\max }$ от ЛИ с длиной волны $\lambda=1,07$ мкм можно проводить при использовании фотоприемного устройства ФПУ1 (рис. 1, поз. 3) и при использовании ФПУ2 (рис. 1, поз. 4). Для проведения измерений в реальных условиях эксплуатации лазерных технологических установок было выбрано ФПУ2.

Результаты измерений облученности диффузно-отраженного и рассеянного Ли от металла при обработке мощным волоконным лазером с подвижным пучком показали проблему узкого эффективного поля зрения ФПУ лД-прибора. Точка (мишень), в которую был направлен целеуказатель (рис. 1, поз. 5), находилась в середине траектории лазерного луча. По мере приближения луча к мишени значения облученности увеличивались. Полученная зависимость облученности (в относи ${ }^{-}$ тельных единицах) от пространственных характеристик (времени) иллюстрирует рис. 2.

Видно, что величина $E_{\max }$ превышает на два порядка текущие значения облученности (рис. 2). Появление максимального значения облученности $E_{\max }$ соответствовало периоду $t=90$ с после старта технологического процесса. Этот момент времени соответствовал пространственному поло-

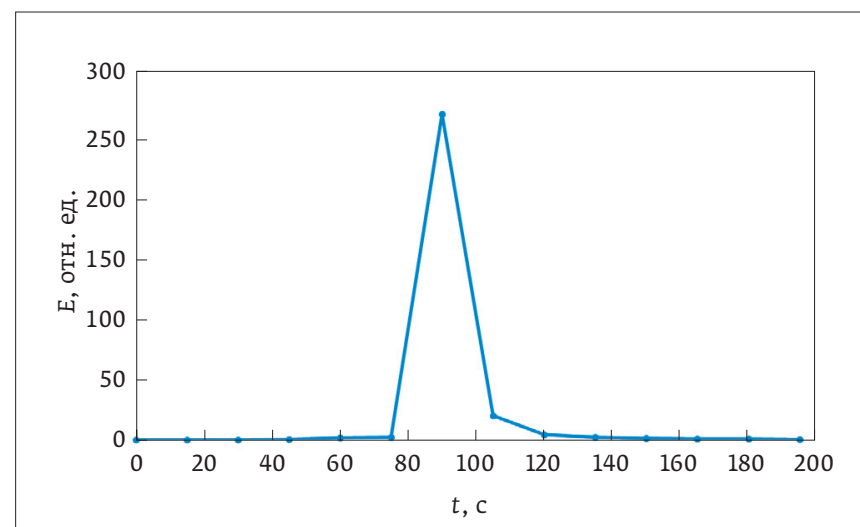

Puс. 2. Зависимость облученности от времени

жению лазерного луча примерно в середине траектории (в мишени).

Для определения угловой характеристики чувствительности фотоприемника ФПУ1 был проведен эксперимент (схема эксперимента показана на рис. 3а), в результате которого были получены зависимости $E_{\max }$ от угла падения лучей на фотоприемник ФПУ1. Результаты измерений значений энергетических характеристик (облученности) Ли от угла падения лучей на фотоприемник ФПУ1 БФП лД относительно оси пучка лазерного излучения представлены на рис. 3b и в таблице. Данные говорят о том, что при отклонении пучка ЛИ от линии нормали на $2,5^{\circ}$ показания облученности изменяются приблизительно в 1,5 раза, а при отклонении на $5^{\circ}$ - примерно в 4 раза. Таким образом, расположение БФП, т.е. выбор точки контроля, оказывает решающее влияние на точность измерений.

\section{ПРИМЕНЕНИЕ СТАНДАРТА}

В документе ГОСТ Р 12.1.031-2010 «ССБТ. Лазеры. Методы дозиметрического контроля лазерного излучения" (ГОСТ Р 2010) определена методика выбора точек контроля. Описан подход к определению границ рабочей зоны и установлению в пределах границы зоны возможного повреждения глаз оператора, а также указано, где следует размещать лд при проведении измерений. Приведены возможные схемы выбора точек контроля для вертикального или горизонтального падения пучка при произвольно ориентированной отражающей поверхности, горизонтальном или вертикальном расположении [2].

При разработке ГОСТ Р 2010 подразумевалось, что лазер - это стационарное устройство с неподвижным сравнительно маломощным пучком, который позволяет проводить выбор точек кон- 


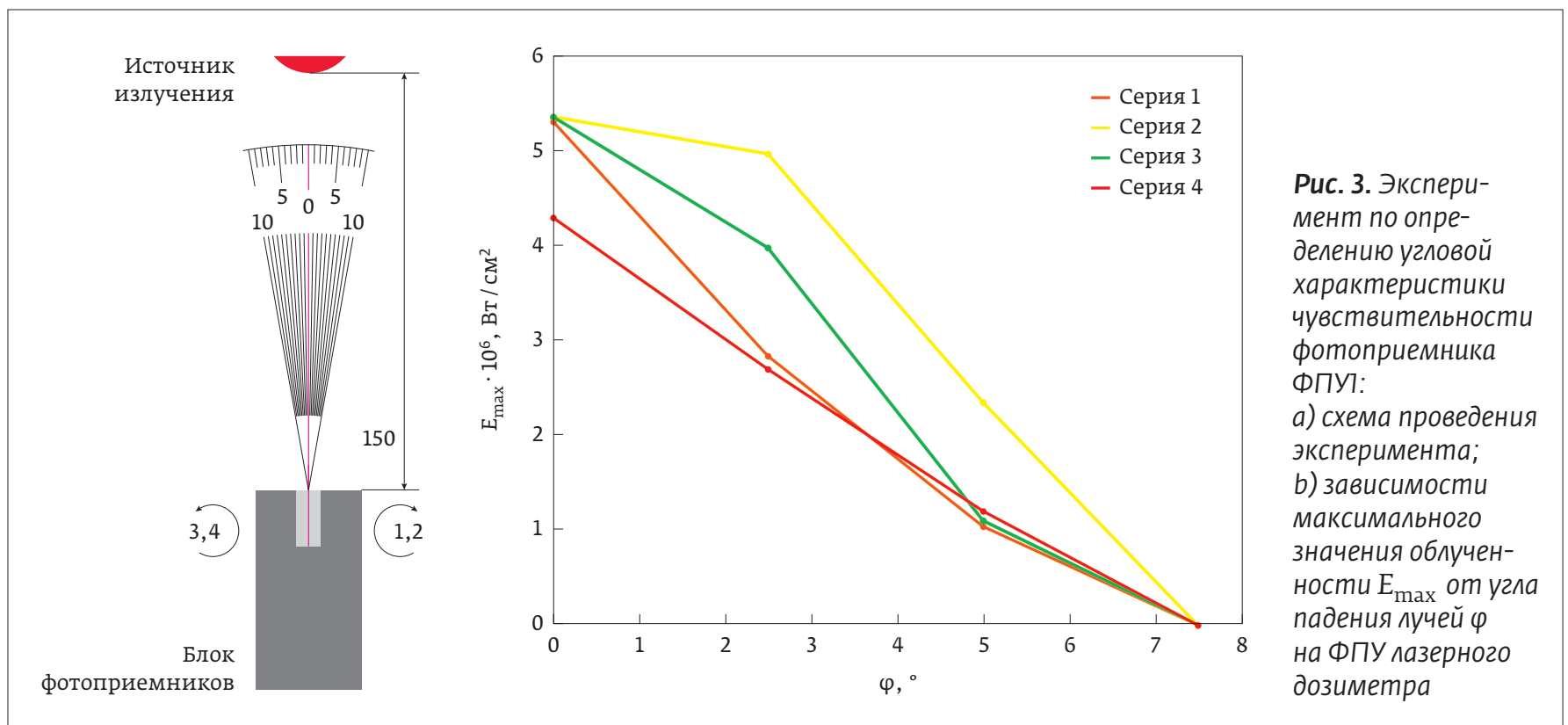

троля, измерения и расчеты относительно неподвижного пучка. Разработчики «ЛД-07» учитывали нормативную документацию (НД) и возможности лазерного оборудования того времени. Эти аспекты и результаты проведенных измерений говорят о том, что правильный выбор точки контроля и получение достоверных значений облученности возможно только при неподвижном положении лазерного пучка.

В современных технологических установках (сварка, резка, наплавка, маркировка и т.д.) пучок подвижен, причем движется в разных плоскостях (находится в разных пространственных положениях в процессах 2D- и 3D-обработки):

Таблица. Результаты эксперимента

\begin{tabular}{|c|c|c|c|c|c|}
\hline \multirow[t]{2}{*}{ № } & \multirow[t]{2}{*}{$\varphi,^{\circ}$} & \multicolumn{4}{|c|}{$E_{\max } \cdot 10^{6}, \mathrm{BT} / \mathrm{CM}^{2}$} \\
\hline & & Серия 1 & Серия 2 & Серия 3 & Серия 4 \\
\hline 1 & 0 & 5,31 & 5,36 & 5,36 & 4,3 \\
\hline 2 & 2,5 & 2,84 & 4,97 & 3,98 & 2,7 \\
\hline 3 & 5 & 1,04 & 2,35 & 1,1 & 1,2 \\
\hline 4 & 7,5 & 0 & 0 & 0 & 0 \\
\hline
\end{tabular}

Измеритель: лазерный дозиметр “ЛД-07». Параметры измерителя: спектральный диапазон 1: 400-1000 нм; спектральный диапазон 2: 1,0-20 мкм; рабочая длина волны: 530 нм; диаметр входной апертуры: $d_{a}=7$ мм. Дополнительные сведения: источник излучения: LED (светодиод); длина волны излучения: 530-535 нм; длительность воздействия: $\Delta t=10$ c; фоновое значение облученности: $\mathrm{E}_{\phi}=0,00 \mathrm{BT} / \mathrm{cm}^{2}$
- пучок перемещается относительно заготовки;

- заготовка перемещается относительно лазерного пучка;

- пучок и заготовка изменяют свои положения друг относительно друга.

В подобных случаях возникают сомнения в наличии теоретических зависимостей для определения оси зеркальных составляющих диффузно-отраженного ЛИ. Выведение эмпирических зависимостей для описания траектории распространения излучения ИК-диапазона без применения специальной аппаратуры затруднительно. Таким образом, представляется невозможным определение точки начала координат, выбор самой системы координат относительно лазерного пучка и проведение дальнейших построений и расчетов. Следовательно, подход, представленный в ГОСТ Р 2010, весьма ограничен и с учетом современных реалий требует доработки и составления рекомендаций для проведения измерений во время штатных режимов работы современных лазерных технологических установок.

Отметим, что диаметры ФПУ (для обоих диапазонов) не соответствуют диаметрам, указанным в СанПинах, по которым проводилось усреднение значений предельно допустимых уровней (Пду) энергетических характеристик ЛИ. Поэтому для определения окончательных значений Пду необходимо проводить дополнительные расчеты. Данный факт не отражается в НД и может быть не учтен при проведении обработки результатов измерений. 


\section{ИЗМЕРЕНИЕ ТЕКУЩИХ ЗНАЧЕНИЙ ОБЛУЧЕННОСТИ РАССЕЯННОГО ИЛИ ОТРАЖЕННОГО ЛИ}

Измерение текущих значений облученности рассеянного или отраженного ЛИ происходит в режиме реального времени, без возможности их фиксации. В результате измерений облученности за время всего технологического цикла регистрируется единственное значение $E_{\max }$, возможно не отражающее реальную картину происходящего процесса.

В случаях единичного производства, при изготовлении уникальных изделий больших габаритов, при технологическом процессе большой длительности могут возникнуть трудности в проведении нескольких серий измерений для определения нескольких значений $E_{\max }$ (это необходимо для обработки результатов измерений в соответствии с метрологическими требованиями). Фиксация текущих значений облученности Ли через равные промежутки времени в течение всего технологического цикла процесса лазерной обработки позволит получить набор максимальных значений $E_{\max }$ за одну серию измерений. Поэтому мы считаем, что для описанных условий наличие данной функции просто необходимо. Подчеркнем тот факт, что в предыдущей версии документа ГОСТ Р 2010 (в ГОСТ 12.1.031-81) данный вопрос был решен. Так пункт П. 3.7 гласил: «При подготовке к контролю непрерывного ЛИ подключают к дозиметру внешний регистрирующий прибор (например, самописец) для записи изменения значений средней мощности (облученности) при изменении времени наблюдения $t^{\prime}$. В текущей версии стан ${ }^{-}$ дарта (ГОСТ Р 2010) данный пункт исключен [3].

\section{ВЫВОДЫ}

Все указанные проблемы говорят о том, что для учета всех ныне существующих особенностей лазерных технологий, корректного проведения измерений облученности ЛИ от подвижного пучка необходим принципиально новый подход в методах дозиметрического контроля ЛИ и усовершенствование прибора, позволяющего удовлетворить все измерительные потребности:

1. Необходимо разработать современные стандарты и создать аттестованные методики измерений ЛИ на рабочих местах операторов лазерных установок, не противоречащие друг другу. Методика измерения должна быть простой, обладать однозначным толкованием и быть понятной для экспертов, которые проводят измерения.

2. После переработки нормативной документации необходимо усовершенствовать ЛД-приборы с учетом внесенных изменений.

Мы предлагаем:

1. Разработать методики дозиметрического контроля Ли, применимые к лазерным технологиям.

2. Предусмотреть в комплектации лД узкополосные светофильтры, которые позволят обеспечить селективность измерений по длине волны.

3. Увеличить эффективное поле зрения ФПУ лД-прибора.

4. Добавить в программное обеспечение лД-прибора функцию фиксации текущих значений измеряемых параметров.

\section{СПИСОК ЛИТЕРАТУРЫ}

1. Руководство по эксплуатации "Дозиметры пазерные «ЛД-07" БВЕК 710000.001 Рэ". ООО «НТМ-Защита".

Rukovodstvo po ekspluatacii "Dozimetry lazernye «LD-07» BVEK 710000.001 RE". 000 "NTM-Zashchita"

2. ГОСТ Р 12.1.031-2010 «ССБТ. Лазеры. Методы дозиметрического контроля пазерного изиучениян.

COST RI2.1.031-2010 "SSBT. Lazery. Metody dozimetricheskogo kontrolya lazernogo izlucheniya".

3. ГОСТ 12.1.031-81 «ССБТ. Методы дозиметрического контроля пазерного изпучения». COST 12.1.031-81 "SSBT. Metody dozimetricheskogo kontrolya lazernogo izlucheniya".

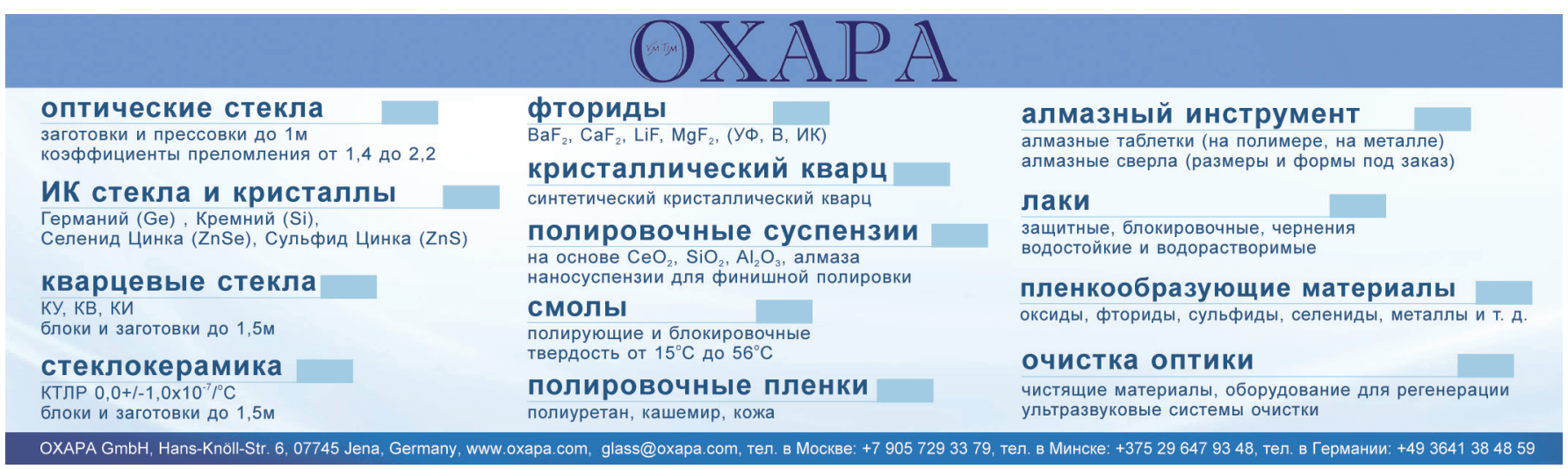

